\title{
Laparoscopic Partial Nephrectomy for Multiple Ipsilateral Renal Tumors Using a Tailored Surgical Approach
}

\author{
Andrew S. Flum, B.S., and J. Stuart Wolf, Jr., M.D.
}

\begin{abstract}
Background and Purpose: Laparoscopic partial nephrectomy (LPN) is safe and effective for solitary renal masses, but its application to multiple ipsilateral renal tumors has been reported infrequently. We review our experience with LPN for multiple ipsilateral renal tumors to assess its role in current practice.

Materials and Methods: We have managed seven patients with multiple ipsilateral renal tumors with LPN. Of the patients, four had an imperative indication for nephron-sparing surgery.

Results: Among the 16 tumors resected, with a mean size of $2.1 \mathrm{~cm}, 9$ (in five patients) were renal cell carcinoma on final pathology. LPN was performed without hilar clamping in four patients (no-clamp group), and with hilar clamping and a sutured bolster in three patients (clamp-suture group). The no-clamp group had a lower mean operative time than the clamp-suture group (185 vs. 225 minutes), similar mean estimated blood loss ( 363 vs. $417 \mathrm{~mL}$ ), and shorter hospital stay (1.8 vs. 3 days). The only complication was an intraoperative hemorrhage necessitating blood transfusion, and there was one focal-positive margin, both in patients in the clamp-suture group. Among the five patients with cancer, there have been no local recurrences or metastases during a mean radiographic follow-up of 48 months.

Conclusions: LPN, with a tailored approach that spares some patients from renal ischemia, appears to be safe and effective in this small series of selected patients with multiple ipsilateral renal tumors.
\end{abstract}

\section{Introduction}

L APAROSCOPIC PARTIAL NEPHRECTOMY (LPN) has become - an accepted surgical option in the management of solitary renal tumors, with efficacy comparable to open surgical nephron-sparing surgery (NSS). ${ }^{1,2}$ The use of this procedure likely will expand as the duration of follow-up in patients undergoing LPN increases ${ }^{3}$ and outcomes similar to those associated with open surgical NSS are demonstrated. ${ }^{4}$ With the application of LPN to the management of small solitary renal tumors, the natural progression is to investigate the limits of the indications for this procedure.

Radical nephrectomy has been the established standard in the treatment of multifocal ipsilateral renal tumors, but various reports have suggested that open NSS may have an equivalent efficacy in selected patients. ${ }^{5,6}$ There have been reports from two institutions of LPN for multiple ipsilateral renal tumors that provide early data on the potential viability of LPN as an approach to treating multifocal ipsilateral disease. $^{7-9}$ We present our experience with managing multiple synchronous ipsilateral renal tumors utilizing our approach to LPN that, as previously described, ${ }^{10,11}$ is tailored to tumor characteristics.

\section{Materials and Methods}

From a database approved by our Institutional Review Board, we identified seven patients who underwent LPN for multiple (two or more) ipsilateral renal masses suspected to be renal cancer. We did not include patients who underwent laparoscopic cryoablation. All patients had undergone preoperative imaging with computed tomography or magnetic resonance imaging. The approach to LPN was transperitoneal standard laparoscopy or hand-assisted laparoscopic surgery (HALS), by a single surgeon (J.S.W.), with resection of tumors under ischemic or nonischemic conditions, and with or without the use of a sutured bolster after tumor resection, as described below.

The procedure was performed using HALS before the development of our tailored approach in two patients, ${ }^{10,11}$ whereas the other five underwent surgery after our treatment selection criteria. Briefly, location of the tumor determines the laparoscopic approach, depth of penetration into the renal parenchyma determines whether hilar clamping is utilized, and proximity of the resection to the renal sinus or collecting system determines management of the tumor bed. Tumors that are posterior (except at the upper pole) are approached

Department of Urology, University of Michigan Health System, Ann Arbor, Michigan. 
retroperitoneoscopically, and for other tumors a transperitoneal standard laparoscopic approach is used except when intraabdominal manual positioning of the kidney would facilitate resection, in which case HALS is used. This latter criterion is subjective, and with the addition of a robot assistance to our armamentarium, it is in flux. Tumors that penetrate less than $5 \mathrm{~mm}$ into the renal parenchyma are resected without hilar clamping, and any deeper tumors are removed after temporary hilar occlusion. Laparoscopic ultrasonography, which was used in all cases in this series, is routinely used to help make this critical assessment. Finally, if the tumor resection enters the renal sinus or collecting system, then a sutured bolster is used for tumor bed management, and in other cases coagulation devices and hemostatic agents are sufficient. We obtain a frozen section of the margin of the mass, or a biopsy of the resection margin on the kidney, only when the gross appearance of the mass suggests inadequate resection. We continue with the procedure, but will re-resect if the frozen section comes back positive.

We do not routinely obtain nuclear medicine renal scans before or after LPN. Perioperative data were collected from our prospectively maintained database, and missing data were gathered from the medical record retrospectively, including operative approach, estimated blood loss (EBL), ischemia time, operative time, blood transfusions, complications, hospital stay, preoperative serum creatinine, serum creatinine at last follow-up, and final pathology data.

\section{Results}

Data for indication, tumor characteristics, operative approach, and pathology are summarized in Table 1. Among the seven patients, a total of 16 tumors were resected -2 tumors in six patients and 4 tumors in one patient. Of the patients, four $(57 \%)$ had an imperative indication for NSS, which included bilateral renal masses, solitary kidney, von HippelLindau syndrome, and recurrent renal calculi. The mean tumor size was $2.1 \mathrm{~cm}$ (median 1.7, range 0.6-4.8). In three patients, at least one of the tumors approached close to or was abutting the renal sinus, whereas the other four patients had peripheral lesions with a depth of penetration into the renal parenchyma of less than $5 \mathrm{~mm}$. The multifocality was known preoperatively in four patients (Fig. 1). In the other three patients the smaller tumor was detected intraoperatively by palpation, observation, and/or intraoperative ultrasonography (Fig. 2).

The initial operative approach in four cases was HALS and standard laparoscopy in the other three cases. Of the four approached with HALS, patients 1 and 2 were early in our experience (before we devised our tailored approach), and patient 2 would be approached with standard laparoscopy today. One case initially approached with standard laparoscopy was converted to HALS because of difficulty dissecting out the kidney from adherent perinephric fat. LPN was conducted without hilar clamping in the four patients with peripheral and shallow masses (no-clamp group). In the three patients with at least one tumor abutting the renal sinus, LPN was conducted under ischemic conditions (clamp-suture group) with complete hilar clamping in two cases and clamping of only a lower pole renal artery in one patient (patient 7). Both renal tumors were resected under ischemic conditions in two patients, and in one patient (patient 6) the

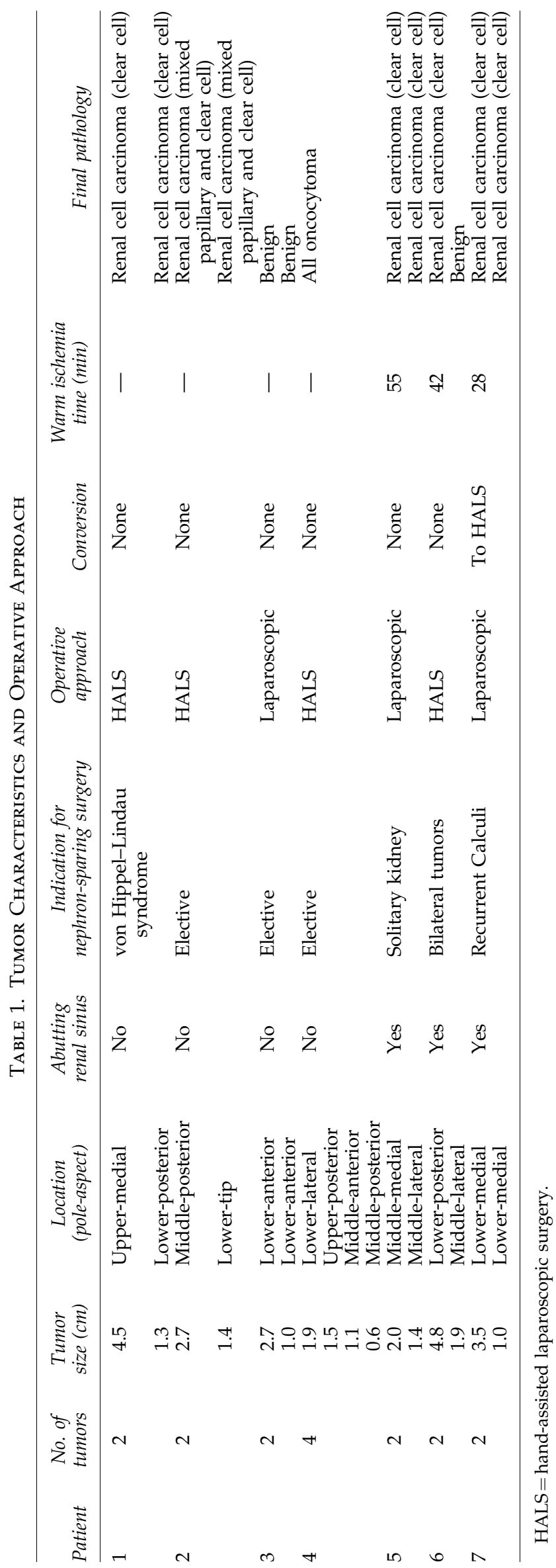




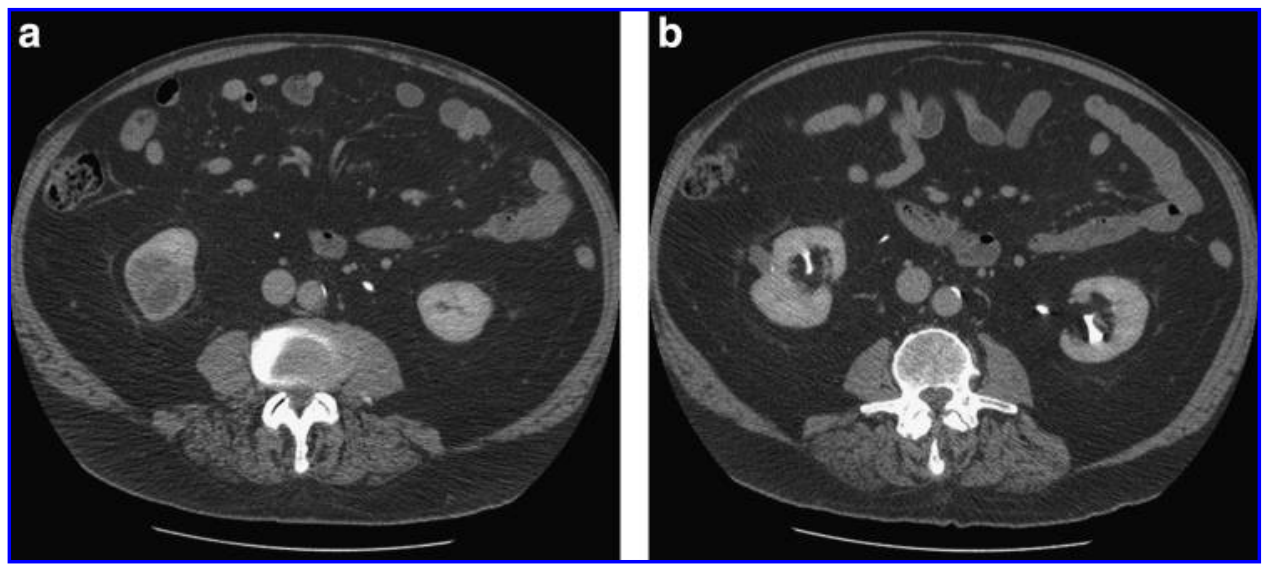

FIG. 1. Computed tomograms, patient 6 . (a) $4.8 \mathrm{~cm}$ lower pole posterior tumor (right kidney). (b) $1.9 \mathrm{~cm}$ midrenal lateral tumor (right kidney).

smaller tumor was resected before clamping for resection of the primary tumor. In the three patients in the clamp-suture group, a sutured bolster was placed into the tumor bed after LPN. In the four no-clamp patients, the tumor bed was managed with gelatin sponge plus fibrin glue, gelatin granules plus thrombin mixture, or argon beam coagulation. In all cases each discrete tumor was resected separately; no tumors were resected en-bloc.

Final pathology revealed that seven (44\%) of the masses were clear cell carcinomas, four (25\%) were oncocytomas, three $(19 \%)$ were benign tissue, and two (13\%) were mixed clear cell and papillary carcinomas. Only one patient had discordant pathology among the resected masses.

Data for perioperative parameters, renal function, and follow-up (divided into the no-clamp and clamp-suture groups) are summarized in Table 2. Overall, the mean EBL was $386 \mathrm{~mL}$ (median 400, range 50-1000), mean operative time was 202 minutes (median 175, range 140-268), and mean hospital stay was 2.3 days (median 2, range 1-4). For the noclamp group, mean EBL was $363 \mathrm{~mL}$, mean operative time was 185 minutes, and mean hospital stay was 1.8 days. Among the three clamp-suture patients, the mean ischemic time was 42 minutes (median 42.3, range 28-55), mean EBL was $417 \mathrm{~mL}$, mean operative time was 225 minutes, and mean hospital stay was 3 days. Only one patient experienced a complication. One patient in the clamp-suture group had an intraoperative hemorrhage of approximately $1 \mathrm{~L}$ of blood and was transfused 1 unit of packed red blood cells in the postoperative period. The hilar clamp malfunctioned, and because the patient had a solitary kidney (patient 5) we completed the procedure rather than dissect the hilum further with suboptimal observation. There were no other complications and no other patients required blood transfusions.

Final pathology revealed a focally positive surgical margin in one tumor (patient 7 , in the clamp-suture group). This tumor also had invasion of the perinephric fat (stage pT3a). All other tumors had negative surgical margins and were stage pT1a or pT1b on final pathology.

The mean preoperative serum creatinine for all patients was $1.0 \mathrm{mg} / \mathrm{dL}$ (median 0.9, range 0.9-1.2). The increase in serum creatinine at last follow-up (mean 43.1 months, median 24.9 , range $10.3-110)$ was minimal $(0.1 \mathrm{mg} / \mathrm{dL}$ for both mean and median). The mean duration of radiographic follow-up for the five patients with malignant tumors on final pathology was 48 months (median 19, range 19-111), and none had local recurrence or metastases.

\section{Discussion}

The incidence of ipsilateral multifocal renal cell carcinoma is not accurately known, but has been suggested to be in the range of $5 \%$ to $25 \% .{ }^{12-14}$ When radical nephrectomy was the

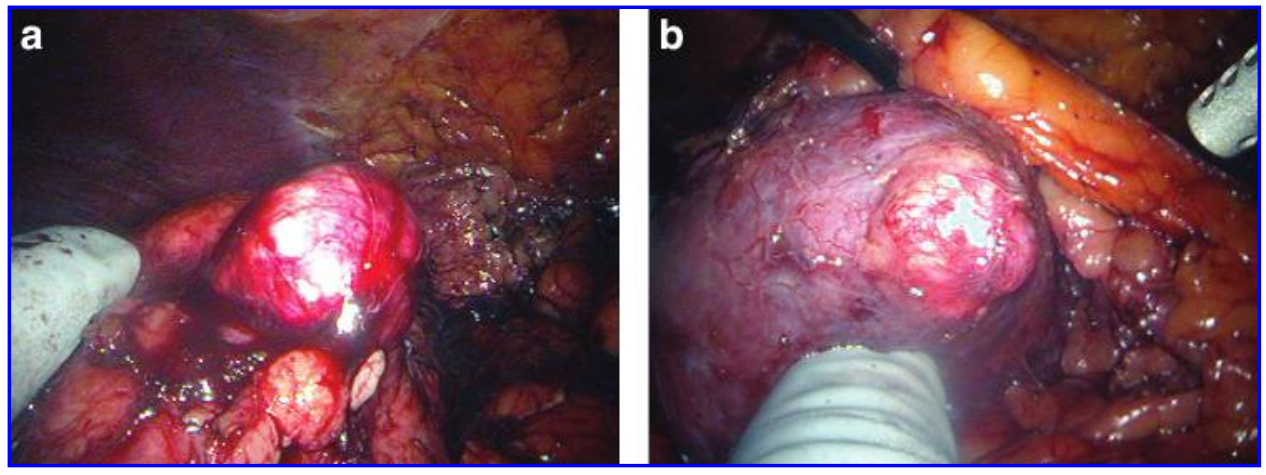

FIG. 2. Intraoperative photographs, patient 2. (a) $2.7 \mathrm{~cm}$ midrenal lateral renal tumor. (b) $1.4 \mathrm{~cm}$ renal tumor at the very tip of lower pole, which was not detected on preoperative computed tomogram with only axial reconstructions (we suspect that the lesion would have been noted preoperatively had coronal reconstructions been obtained). 
Table 2. Perioperative, Renal Function, and Follow-Up Data for laparoscopic Partial Nephrectomy without Clamp or Suture Versus with Clamp and Suture

\begin{tabular}{lccc}
\hline & $\begin{array}{c}\text { No clamp or suture } \\
(\mathrm{n}=4)\end{array}$ & $\begin{array}{c}\text { Clamp and suture } \\
(\mathrm{n}=3)\end{array}$ & $\begin{array}{c}\text { Total } \\
(\mathrm{n}=7)\end{array}$ \\
\hline Mean estimated blood loss, mL (range) & $363(50-600)$ & $417(100-1000)$ \\
Mean ischemic time, minutes (range) & - & $42(28-55)$ & $386(50-1000)$ \\
Mean operative time, minutes (range) & $185(140-268)$ & $225(174-268)$ & $202(140-268)$ \\
No. of blood transfusions (\%) & $0(0)$ & $1(33)$ & $1(14)$ \\
No. of complications (\%) & $0(0)$ & $1(33)$ & $1(14)$ \\
No. of positive margin (\%) & $0(0)$ & $1(33)$ & $1(14)$ \\
Mean hospital stay, days (range) & $1.8(1-2)$ & $3(2-4)$ & $2.3(1-4)$ \\
Mean preoperative serum creatinine, mg/dL (range) & $1.1(0.9-1.2)$ & $1(0.9-1.2)$ & $1(0.9-1.2)$ \\
Mean serum creatinine at last follow-up, mg/dL (range) & $1.1(0.9-1.4)$ & $1.1(1.0-1.2)$ & $1.1(0.9-1.4)$ \\
Mean duration of contact, months (range) & $60.7(10.4-112)$ & $21(18.7-25.3)$ & $43.6(10.4-112)$ \\
Mean duration of radiographic follow-up, months (range) & - & - & $48.9(18.7-112)$ \\
\hline
\end{tabular}

${ }^{a}$ Excludes the two patients with only benign lesions.

standard of care for localized tumors, multifocality was not a concern. With the demonstrated efficacy of open surgical $\mathrm{NSS},{ }^{15}$ and more recently laparoscopic NSS, ${ }^{3}$ for small solitary renal tumors with or without a normal contralateral kidney, the consideration of multifocal disease becomes more important. There is already good evidence that open NSS can be applied to the treatment of multiple ipsilateral tumors, ${ }^{5,6}$ but less is known about LPN for this indication, with the results in only 24 patients published to date. ${ }^{7-9}$

With the increasing utilization of LPN, incidental multifocal disease discovered at the time of planned NSS (i.e., not detected preoperatively) is another important consideration. Gohji and associates ${ }^{16}$ examined the radical nephrectomy specimens of patients who would have met the criteria for NSS and found a $15.6 \%$ rate of incidental multifocal renal cell carcinoma. Although many of these incidentally discovered foci were very small and of unclear clinical significance, it does suggest that as experience with LPN is gained there will be more cases (just as in open surgical NSS) where an additional tumor is found at the time of intended LPN for a solitary lesion (as was the case in three of our cases).

Since the literature on the topic of LPN for multifocal tumors is limited, even our small series adds useful information. Most importantly, there were no local or metastatic recurrences at a mean radiographic follow-up exceeding 4 years, with the greatest duration of follow-up being 112 months. The one patient with a focal-positive margin has 19 months of radiographic follow-up without evidence of recurrence to date. Additionally, renal function has been well maintained in all patients. Our results are similar to those in the three (two of which are overlapping cohorts from the same institution) published series of LPN for multifocal tumors from the same institution, which reported on 14 patients with a median follow-up of 38.5 months and no tumor recurrences ${ }^{7,8}$ and 10 patients with robotic assistance to LPN (one converted to open surgery). ${ }^{9}$

We cannot make any firm conclusions on the utility of our tailored approach to LPN for multiple ipsilateral tumors owing to the small size of our series and the short follow-up, but our results suggest that, so far, the tailored approach to LPN appears as applicable in this setting as it does in the setting of LPN for solitary tumors. The goal of reducing ischemia time is very pertinent when addressing multiple ipsilateral renal tumors. Mean ischemia time for patients with solitary renal tumors undergoing LPN with clamping and suturing was 30.3 minutes in our overall experience using the tailored approach, ${ }^{11}$ whereas the mean ischemia time for the clamp-suture group in this series of LPN for multiple tumors was 42 minutes. With the longer resection and reconstruction times that would be expected for multiple as opposed to solitary tumors, hilar clamping and ischemia time would be correspondingly longer as well-so sparing the hilar clamping in patients with shallow tumors that do not approach the renal sinus or collecting system is even more attractive.

Patient selection is paramount in improving the likelihood of a good outcome of LPN for multiple renal masses. Although most partial nephrectomies for small renal masses at our institution currently are performed laparoscopically, ${ }^{17}$ and we apply a wide range of approaches to enhance our ability to provide minimally invasive resection of renal tumors, including hand and robotic assistance, there are still some small renal masses that are best approached with open surgery. We have provided an overview of our selection criteria for our various approaches to LPN, but we cannot provide a similar template for the selection of laparoscopic versus open surgical partial nephrectomy; that decision is based upon the surgeon's own experience.

\section{Conclusions}

LPN appears to be a safe and effective treatment for patients with multiple ipsilateral renal tumors in this small series. Further characterization of the outcomes of LPN in the setting of multifocal disease is important to assist with the difficult management decisions that arise in patients with incidentally discovered multifocality at time of planned NSS, and in patients with multifocal disease in the setting of an elective indication for NSS. A tailored approach to LPN based on tumor characteristics, which allows renal ischemia to be avoided in select patients, appears to be a reasonable strategy in the treatment of multiple ipsilateral renal tumors.

\section{Disclosure Statement}

Andrew S. Flum, B.S.- - none. 
J. Stuart Wolf, Jr., M.D.-Terumo Corporation (consultant), Gyrus-ACMI (lecturer).

\section{References}

1. Gill IS, Matin SF, Desai MM, et al. Comparative analysis of laparoscopic versus open partial nephrectomy for renal tumors in 200 patients. J Urol 2003;170:64-68.

2. Seifman BD, Hollenbeck BK, Wolf JS Jr. Laparoscopic nephron-sparing surgery for a renal mass: 1-year minimum follow-up. J Endourol 2004;18:783-786.

3. Lane BR, Gill IS. 5-Year outcomes of laparoscopic partial nephrectomy. I Urol 2007;177:70-74.

4. Gill IS, Kavoussi LR, Lane BR, et al. Comparison of 1,800 laparoscopic and open partial nephrectomies for single renal tumors. J Urol 2007;178:41-46.

5. Krambeck AE, Iwaszko M, Leibovich BC, et al. Long-term outcome of multiple ipsilateral renal tumours found at the time of planned nephron-sparing surgery. BJU Int 2008;101: 1375-1379.

6. Minervini A, Serni S, Giubilei G, et al. Multiple ipsilateral renal tumors: Retrospective analysis of surgical and oncological results of tumor enucleation vs radical nephrectomy. Eur J Surg Oncol 2009;35:521-526.

7. Steinberg AP, Kilciler M, Abreu SC, et al. Laparoscopic nephron-sparing surgery for two or more ipsilateral renal tumors. Urology 2004;64:255-258.

8. Lin YC, Turna B, Frota $R$, et al. Laparoscopic partial nephrectomy versus laparoscopic cryoablation for multiple ipsilateral renal tumors. Eur Urol 2008;53:1210-1216.

9. Boris $\mathrm{R}$, Proano $\mathrm{M}$, Linehan $\mathrm{WM}$, et al. Initial experience with robot assisted partial nephrectomy for multiple renal masses. J Urol 2009;182:1280-1286.

10. Johnston WK III, Montgomery JS, Seifman BD, et al. Fibrin glue v sutured bolster: Lessons learned during 100 laparoscopic partial nephrectomies. J Urol 2005;174:47-52.

11. Weizer AZ, Gilbert SM, Roberts WW, et al. Tailoring technique of laparoscopic partial nephrectomy to tumor characteristics. J Urol 2008;180:1273-1278.
12. Jacqmin D, Saussine CT, Roca D, et al. Multiple tumors in the same kidney: Incidence and therapeutic implications. Eur Urol 1992;21:32-34.

13. Kletscher BA, Qian J, Bostwick DG, et al. Prospective analysis of multifocality in renal cell carcinoma: Influence of histological pattern, grade, number, size, volume and deoxyribonucleic acid ploidy. J Urol 1995;153:904-906.

14. Dimarco DS, Lohse CM, Zincke H, et al. Long-term survival of patients with unilateral sporadic multifocal renal cell carcinoma according to histologic subtype compared with patients with solitary tumors after radical nephrectomy. Urology 2004;64:462-467.

15. Fergany AF, Hafez KS, Novick AC. Long-term results of nephron sparing surgery for localized renal cell carcinoma: 10-year follow-up. J Urol 2000;163:442-445.

16. Gohji K, Hara I, Gotoh A, et al. Multifocal renal cell carcinoma in Japanese patients with tumors with maximal diameters of $50 \mathrm{~mm}$ or less. I Urol 1998;159:1144-1147.

17. Hollingsworth JM, Miller DC, Dunn RL, et al. Surgical management of low stage renal cell carcinoma: Technology does not supersede biology. Urology 2006;67:1175-1180.

Address correspondence to: J. Stuart Wolf, Jr., M.D. Department of Urology University of Michigan Health System 1500 East Medical Center Drive, TC 3875 Ann Arbor, MI 48109-5330

E-mail: wolfs@umich.edu

$\begin{aligned} & \text { Abbreviations Used } \\ \mathrm{EBL} & =\text { estimated blood loss } \\ \mathrm{HALS} & =\text { hand-assisted laparoscopic surgery } \\ \mathrm{LPN} & =\text { laparoscopic partial nephrectomy } \\ \mathrm{NSS} & =\text { nephron-sparing surgery }\end{aligned}$



This article has been cited by:

1. Alexander Tsivian, Matvey Tsivian, Shalva Benjamin, A. Ami Sidi. 2011. Laparoscopic partial nephrectomy for multiple tumours: feasibility and analysis of peri-operative outcomes. BJU International no-no. [CrossRef] 